\title{
Management of Co-Morbidity of Depression and Chronic Non- Communicable Diseases in Rwanda
}

\author{
Madeleine Mukeshimana ${ }^{1,2}$, Gugu Mchunu ${ }^{2 *}$
}

\section{OPEN ACCESS}

Citation: Madeleine Mukeshimana, Gugu Mchunu. Management of Co-Morbidity of Depression and Chronic NonCommunicable Diseases in Rwanda. Ethiop J Health Sci 2017;27(1):17-26. doi:

http://dx.doi.org/10.4314/ejhs.v27i1.4

Received: July 14, 2016

Accepted: September 15, 2016

Published: January 1, 2017

Copyright: Madeleine M, et al. This is an open access article distributed under the terms of the Creative Commons

Attribution License, which permits unrestricted use, distribution, and reproduction in any medium, provided the original author and source are credited.

Funding: Rwanda Education Board

Competing Interests: The authors

declare that this manuscript was approved

by all authors in its form and that no

competing interest exists.

Affiliation and Correspondence:

${ }^{1}$ College of Medicine and Health

Sciences, University of Rwanda, Rwanda

${ }^{2}$ Howard College, University of KwaZulu Natal, South Africa

*Email: mchunug@ukzn.ac.za

\section{ABSTRACT}

BACKGROUND: Chronic non-communicable diseases (NCDs) are a major global health problem of the 21 stcentury. They are now the world's leading cause of disease burden and high mortality. An even more alarming health problem is when depression coexists with chronic NCDs, as is frequently the case. Management of this co-morbidity with collaborative care has become a global topic of interest, with the World Health Organization (WHO) recommending implementation of collaborative care for this purpose. The study investigated existing protocols and/or interventions for managing this co-morbidity in Rwandan district hospitals.

METHODS: The study used an action research design involving a research team of 14 health care professionals to collaboratively identify existing protocols or interventions for managing comorbidity of depression and NCDs in Rwanda. Focus group discussion using a structured interview guide was used to collect qualitative data, followed by qualitative content analysis using inductive approach.

RESULTS: We found no particular protocols or interventions in place to manage the co-morbidity of depression and chronic NCDs. Depression and chronic NCDs were found to be treated separately, in separate health care settings and by different health professionals.

CONCLUSION:The findings revealed a gap in management of comorbid depression and chronic NCDs in Rwanda district hospitals. We recommend that health care providers follow the WHO collaborative care advisory for better quality care and better patient improvement in management of this co-morbidity.

KEYWORDS: Chronic NCDs,depression, co-morbidity, management 


\section{INTRODUCTION}

Chronic NCDs are a major global health problem, causing morbidity and mortality in all countries(1$3)$. More alarming still is when depression coexists with chronic NCDs (as is frequently the case),which is associated with major health consequences including high morbidity and mortality (4-9). Chronic NCDs that are frequently linked with depression include cardiovascular diseases, diabetes, cancer and chronic respiratory diseases (10-12). This co-morbidity has become a major concern worldwide, and optimal management of the problem is increasingly a topic of interest. A number of studies that have reviewed the management of this co-morbidity in various settings confirm that collaborative care is cost-effective in management of the co-morbidity (13-15). Collaborative care for management of comorbid depression and NCDs in primary care settings is also recommended by the WHO (16). In low-income countries, it is, however, still frequently the case that mental health care is separate from physical care (17). This separation of treatment is associated with significant negative health effects such as complex medication regimens with high risk of drug interactions and poor medication adherence, duplicative medical tests, unnecessary hospitalizations and increased mortality (13-15).

The co-morbidity of depression and chronic NCDs in Rwanda is an ongoing concern for health care providers, with estimated prevalence of depression among diabetic and hypertensive patients,for example, being $27 \%$ and $29 \%$ respectively (18). Patients with chronic NCDs in Rwanda also face numerous difficulties in addition to the daily burden of managing their illnesses and their complications-difficulties common to patients from a developing country such as shortage of health care professionals, long distances to the health care settings and socioeconomic problems. Likewise, some of these patients have also been victims of the 1994 genocide in Rwanda: orphans, widows, the unemployed, or simply those who have no one to care for them. All these difficulties make patients from Rwanda with chronic NCDs vulnerable to stress, anxiety and depression.
The focus of this study was to investigate the current situation regarding management of comorbidity of depression and chromic NCDs in Rwanda district hospitals. It therefore aimed to indicate potential solutions to deficiencies that emerge.

\section{METHODS}

This article is part of a larger study focussed on adapting the collaborative care model to the Rwandan context for management of co-morbid of depression and chronic NCDs. The study was ethically approved by the University of KwaZuluNatal Ethics Committee, the University of Rwanda Institutional Review Board and the Rwanda Ministry of Health.

Action research sequential-explanatory design with mixed methods was used for the larger study. The study was conducted in three cycles adapted from action research procedures (19). In the first cycle which was exploratory, the researcher identified the problem by exploring the prevalence of depression among diabetic and hypertensive patients and also the availability of protocol/interventions to manage this co morbidity. For this first cycle, mixed methods approach (Quantitative and qualitative) was used where quantitative approach was used to determine the prevalence of depression among participants with chronic NCDs represented by diabetes and hypertension in our study. While qualitative approach was used to identify existing protocol/interventions to manage this co morbidity. Therefore, this article presents the findings from the qualitative aspect which explored the current situation regarding management of the co-morbidity of depression and chronic NCDs in Rwanda district hospitals.

The research areas were three selected district hospitals in Rwanda. The two first hospitals were district hospitals from urban area with endocrinology and mental health services, and the third hospital was a district hospital from a rural area with endocrinology and mental health services. The selection of hospitals from urban area and one from rural area increased the chance of sample representation from both urban and rural settings.

DOI: http://dx.doi.org/10.4314/ejhs.v27i1.4 
In action research, researchers and health care practitioners come together to identify a problem and propose solutions (20). Accordingly, a research team of 14 health professionals (two psychiatrists, three medical doctors, three registered nurses, three chief nurses and three mental health nurses) participated in our study. Simple random sampling was used to choose the team members. They were all working in three participating hospitals except for two psychiatrists who were working in other settings (in Rwanda there are no psychiatrists based in district hospitals). The inclusion criteria for the medical doctors and registered nurses was that they were already working with patients with chronic NCDs.

Focus group discussion was used as a method of data collection. The focus group was held at the University of Rwanda, precisely at the College of Medicine and Health Sciences. Before the discussion took place, the researcher provided more information about the purpose of the study and asked the team members who agreed to participate in the study to sign the informed consents . Interviews conducted by the researcher followed the key questions on the structured interview guide, with a range of answers from the various team members on each question. The researcher played a facilitating role, making sure that the group focused on the problem being explored and encouraging participation by every team member. Audio tape and note-taking were used, and the research assistant (a registered nurse working in the Department of Nursing at the University of Rwanda) helped in the note-taking. The focus group meeting lasted for two hours.

Content analysis of qualitative data was done using inductive approach. Data analysis by was conducted in three phases, as outlined by Elo and Kyngäs(21): a preparation phase, an organizing phase and a reporting phase. In the preparation phase, data was read and reread, and units of analysis were chosen. In the organizing phase, categories and sub-categories were developed, while in the reporting phase, results were reported.

Research ethics was respected as the study involved human beings. Care was taken at each stage on permission to conduct the study and respondents' right to self-determination, privacy, anonymity, confidentiality, fair treatment and protection from harm and discomfort were observed. All participants signed the informed consent form.

Table 1: Profile of the research team members

\begin{tabular}{lllll}
\hline $\begin{array}{l}\text { Participant } \\
\text { code }\end{array}$ & Gender & Position & Institution & Experience \\
01 & Male & Psychiatrist & Psychiatric referral hospital & 13 years \\
02 & Male & Psychiatrist & Ministry of Health & 10 years \\
03 & Male & Medical doctor & Hospital no. 1 & 5 years \\
04 & Male & Medical doctor & Hospital no. 2 & 6 years \\
05 & Male & Medical doctor & Hospital no. 3 & 3 years \\
06 & Female & Chief of nursing & Hospital no1 & 4 years \\
07 & Female & Chief of nursing & Hospital no. 2 & 6 years \\
08 & Female & Chief of nursing & Hospital no. 3 & 8 years \\
09 & Female & Registered nurse & Hospital no. 1 & 3 years \\
10 & Female & Registered nurse & Hospital no. 2 & 6 years \\
11 & Female & Registered nurse & Hospital no. 3 & 4 years \\
12 & Female & Mental health nurse & Hospital no. 1 & 6 years \\
13 & Female & Mental health nurse & Hospital no. 2 & 8 years \\
14 & Female & Mental health nurse & Hospital no. 3 & 4 years \\
\hline
\end{tabular}




\section{RESULTS}

Categories and sub-categories emerging from questions exploring the current management of co-morbidity

\section{Categories emerged from our analysis of} data:

- meaning of co-morbidity;

- common co-morbidities;

- awareness of co-morbid depression with chronic NCDs;

- common NCDs associated with depression

- screening tools of depression in patients with NCDs;

- particular protocol or interventions to the management of co-morbidity of depression and NCDs.

Meaning of co-morbidity: Participants offered various definitions which nonetheless which correlated with one another; all were in agreement that co-morbidity of illnesses is the occurrence of more than one disease in the same person.

Common co-morbidities in the district hospitals: When asked about common comorbidities that they had to deal with in their respective hospitals, most participants cited diabetes and hypertension. Also, frequently cited by the participants were co-morbidities not just of two diseases but also of three diseases (e.g. diabetes, hypertension and gastritis; diabetes, hypertension and asthma; diabetes, hypertension and depression).

Awareness about co-morbid depression with chronic NCDs: Participants' responses when asked if they were aware of this co-morbidity were grouped, following analysis, into five subcategories:

- awareness of occurrence of the comorbidity;

- non-focus on depression;

- suspected by some signs and symptoms;

- referral of the patient if suspected to the mental unit;

- no follow up of the patient.

\section{Awareness of occurrence of the co-morbidity}

All respondents revealed that they knew very well that depression can occur any time in a patient with one or more chronic NCDs. One participant said, "It is obvious, once you have a chronic disease, you have so many questions in your head, am I going to die? Till when will, I take these drugs? Sometimes, you don't even eat, your lose appetite, then puff, depression is there, and you can't help it'.

Non-focus on depression: Although all participants acknowledged that depression may often occur in patients with chronic NCDs, they also agreed that depression in patients with NCDs is often unrecognized by health professionals or simply not there is remembered. One participant said, "We are aware of that comorbidity but most of time we don't pay attention to it, mostly we focus on medical problem. Depression will be the last thing to think about". Another comment was, "Most time we forget about depression you know it is not our domain".

A psychiatric explained that if a patient with both depression and one or more than one chronic NCDs happens to visit the hospital or be hospitalized, he will in that case be referred to the general hospital to get treatment for the medical problem and then come back for the mental problem.

Participants also explained that patients may not even recognize signs and symptoms of depression, and this may cause that most of them Don't consult for depression, since they are unware that they may have it.

Suspected from signs and symptoms: Participants explained that although depression is mostly unrecognized in patients with NCDs, with its signs and symptoms often being confused with those of the medical problem, there are some signs and symptoms which trigger suspicion of depression in a patient. One participants commented, "Depression will be the last thing to think about unless depression is too much, like when you receive a client who is crying and you try to find out a medical problem causing those tears and you don't find it". That time you may suspect about depression".

When the researcher asked to know if all participants would suspect depression from certain signs and symptoms, some responded that it was not usually the case. One participant said, "Sometimes you don't see it, it is like you 
are blind and your mind is on the medical problem".

Referral of the patient to the mental unit without follow-up: The researcher wanted to know what happened when a doctor suspected depression in a patient. Did he/she let the patient continue like that or was something done? Participants answered that when a medical doctor suspects depression while caring for a patient, he treats the medical problem, and then refers the patient to the mental unit. The responses indicates that this referral would not be official but just advice to the patient. One participant said, "Oh, this transfer is not an official one, it is just advising the patient to go and see the mental nurse and they are no documents used and no follow up."

Common chronic NCDs associated with depression: Cardiac diseases, diabetes and asthma were the diseases which participants reported as those most frequentlyassociated with depression in their hospitals. One participant commented as follows:

“... because patients with those diseases are always stressed, wondering if they will live tomorrow or next tomorrow.

.. Let's take diabetes, patients with diabetes always say "I fear that I may die of hyperglycaemia or hypoglycaemia any time of the day ..see people with cardiac diseases: "Doctor I fear that I sleep and go..., I die because of my heart.

..imagine people with asthma: "Doctor, imagine if I forgot my inhaler with salbutamol, I don't want to think what will happen to me.
..Imagine these people living in every day fear, this is what called depression ".

Screening tools of depression in patients with NCDs: Tools to screen for depression such as Patient Health Questionnaire-9 (PHQ-9) and Beck Depression Inventory (BDI) exist in district hospitals, and mental nurses use them every day to screen for depression. The surprise, however, was that mental nurses were not aware that these tools are also highly recommended for screening for depression among patients with chronic NCDs, PHQ-9 in particular(15).

\section{Particular protocols orinterventions to manage co-morbidity of depression and NCDs}

No particular protocols or specific interventions: All participants confirmed that no particular protocols or interventions existed to manage this co-morbidity. One participant said, "I don't think we do have such protocol or interventions in our hospital. You know as we said earlier, if a medical doctor thinks that this person may have depression, then he/she will ask him/her to go to the mental unit. It is that all'.

Separate treatment by separate health professionals: The participants made it clear that for them depression and NCDs are different diseases, treated separately and by different health professionals in their hospitals. One of the participants said, "Depression is treated in mental units by mental professionals and NCDs are treated in medical units by medical professionals. We may also facilitate the transfer, but really we do not go beyond".

No Follow up: It was clear from the discussion that no follow-up was done by medical professionals once they had asked the patient to see the mental professionals.

Table 2: Categories and sub-categories emerging from questions on management of co-morbidity in Rwanda 


\begin{tabular}{ll}
\hline Categories & Sub-Categories \\
\hline Meaning of co-morbidities & More than one disease in the same person \\
& Occurrence of a disease from another one in a person \\
Common co-morbidities & Diabetes and hypertension \\
& Diabetes, hypertension and gastritis \\
& Diabetes, hypertension and asthma \\
& Diabetes, hypertension and depression \\
& Renal failure and depression \\
Awareness of co-morbid depression with & Awareness of the occurrence of depression in chronic NCDs \\
chronic NCDs & Non-focus on depression \\
& Suspected by some signs and symptoms \\
& Referral of the patient to the mental unit \\
Common chronic NCDs associated with & No follow up \\
& Cardiac diseases \\
depression & Diabetes \\
& Asthma \\
& Renal diseases \\
Screening tools of depression in patients & Gastritis \\
with chronic NCDs & No tools \\
& PHQ-9 to screen depression in Patients with HIV+ \\
& PHQ-9,BDI in psychiatric hospitals to screen depression in \\
Particular protocol & general \\
Interventions to manage the co- & No particular protocol \\
morbidity of depression and chronic & No specific interventions \\
NCDs & Referral in the mental unit \\
& Separate treatment \\
& No follow up \\
\hline &
\end{tabular}

Table 3: Protocol and interventions to manage co-morbidity of depression and chronic NCDs in Rwanda district hospitals

\begin{tabular}{|c|c|c|c|c|c|c|}
\hline \multirow[b]{2}{*}{$\begin{array}{l}\text { Protocol } \\
\text { Specific protocol } \\
\text { to manage co- } \\
\text { morbiddepression } \\
\text { and chronic } \\
\text { NCDs }\end{array}$} & \multicolumn{6}{|c|}{ Interventions } \\
\hline & $\begin{array}{l}\text { Specific } \\
\text { interventions to } \\
\text { manage co- } \\
\text { morbiddepression } \\
\text { and chronic } \\
\text { NCDs }\end{array}$ & $\begin{array}{l}\text { What is } \\
\text { being done }\end{array}$ & $\begin{array}{l}\text { Who is } \\
\text { involved }\end{array}$ & $\begin{array}{l}\text { Available } \\
\text { resources }\end{array}$ & Outcome & $\begin{array}{l}\text { Client's } \\
\text { satisfaction }\end{array}$ \\
\hline $\begin{array}{l}\text { No specific } \\
\text { protocol }\end{array}$ & $\begin{array}{l}\text { No specific } \\
\text { interventions to } \\
\text { manage the co- } \\
\text { morbidity }\end{array}$ & $\begin{array}{l}\text { Separate } \\
\text { treatment by } \\
\text { separate } \\
\text { health care } \\
\text { professionals } \\
\text { No follow- } \\
\text { up }\end{array}$ & $\begin{array}{l}\text { Medical } \\
\text { doctors } \\
\text { Mental } \\
\text { nurses }\end{array}$ & $\begin{array}{l}\text { PHQ-9 } \\
\text { depression } \\
\text { screening } \\
\text { tool at } \\
\text { district } \\
\text { hospital } \\
\text { PHQ-9 and } \\
\text { BDI at } \\
\text { referral } \\
\text { psychiatric } \\
\text { hospital }\end{array}$ & $\begin{array}{l}\text { Separate } \\
\text { treatment of } \\
\text { both } \\
\text { depression } \\
\text { and medical } \\
\text { condition } \\
\text { Lack of } \\
\text { depression } \\
\text { monitoring }\end{array}$ & $\begin{array}{l}\text { Lack of } \\
\text { information } \\
\text { about } \\
\text { client's } \\
\text { satisfaction } \\
\text { due the lack } \\
\text { of follow up }\end{array}$ \\
\hline $\mathbf{N}$ & & & chro & $\begin{array}{l}\text { ess of } \\
\text { NCDs }\end{array}$ & $\begin{array}{l}\text { norbid de } \\
\text { ng health }\end{array}$ & $\begin{array}{l}\text { ession and } \\
\text { rofessionals }\end{array}$ \\
\hline
\end{tabular}

DOI: http://dx.doi.org/10.4314/ejhs.v27i1.4 
(research team), and frequent NCDs associated with depression

The study found that the health care professionals were aware that depression can occur at any time in a patient with one or more chronic illnesses because of stress, fear or anger resulting from chronic illnesses. However, almost all the health professionals agreed that depression is mostly unrecognized in patients with chronic illnesses, either because the focus is on the medical condition or simply because of confusion between medical signs and depression signs. These findings correspond with those confirmed by other authors. According to Simon (22), the presence of a chronic medical illness may reduce the possibility that physicians or other health care providers recognize or treat depression. Studies have also confirmed that depression is under-diagnosed and under-treated close to $50 \%$ to $65 \%$ of the time in primary health care settings (23-26).

The study found that diabetes, hypertension and asthma were the conditions most reported by health professionals as associated with depression. They explained that these diseases are regarded by most patients as portending imminent death. These qualitative findings were confirmed by quantitative findings of this study that found out tha tdepression was higher in patients with diabetes and hypertension $(83.8 \%)$. These findings confirm those from a systematic review of 31 studies in which higher numbers for depression were found in certain chronic illnesses thatincluded diabetes, asthma and cardiovascular diseases (27). Polsky et al.(28) also reported that lung diseases and heart diseases are among those most associated with depressive patients. Other diseases reported by Kang et al. (29) as particularly associated with depression included cancer, stroke and acute coronary syndrome.

\section{Screening tools to diagnose depression among patients with chronic NCDs}

The findings revealed that in medical units at the district level, there were no specific screening tools to diagnose depression among patients with NCDs. Although the PHQ-9 questionnaire was available in mental health units of these hospitals, mental nurses had been trained by the Ministry of Health to use this tool to screen for depression only in patients with HIV/AIDS. Unfotenately the same nurses were not aware that the tool can be also used to screen for depression among patients with NCDs. Its use is in fact recommended by the WHO for this specific purpose (15). We also found that the BDI was available at the psychiatric referral hospital for screening for depression in general. The tools most recommended by researchers for use in screening for depression among patients with NCDs are PHQ-9 and BDI (15, 30-32).

Our findings suggested non-availability for medical staff at district level of depression screening tools for use among patients with NCDs. Screening tools should made available to medical doctors who should also receive training on how to use these tools to screen for depression among patients with NCDs. Early diagnosis of depression in patients with chronic illnesses is widely supported and recommended $(15,33,34)$.

\section{Protocols or interventions to manage co- morbidity of depression and NCDs}

The study found that no specific protocols or interventions were in place for managing comorbidity of depression and chronic NCDs in the participating district hospitals. When comorbidity was present, it was handled through separate treatments by separate medical health and mental health professionals. There was no collaboration between these health care providers in treating this co-morbidity, and there was no follow-up from medical doctors who may have advised the patient to consult in the mental unit. This separated treatment of comorbid depression and chronic NCDs is not unique to Rwanda and is encountered in most developing countries, as has been shown by Clarke and Currie (17).

Despite WHO's recommendation of collaborative management of this co-morbidity between mental and medical professionals (13), separated treatment persists (17).

This separate treatment continues to be associated with health complications such as complex medication regimens with high risk of drug interactions and poor medication adherence, duplicative medical tests, unnecessary hospitalizations, and worsening mortality (13-15). 
An important point here is that in Rwanda there is decentralized care of chronic NCDs at the primary level (district level) in a program known as "Integration of human resources for chronic care" in which a group of health professionals (4-10 generalist physicians, 2-3 chronic NCD nurses, 4-5 HIV and TB nurses and 2-3 neuropsychiatric nurses) are assigned to the district hospitals to care for chronic illnesses. Based on our findings, however, despite this integration of human resources, there is no evident collaborative strategy between these professionals and cases are seen and treated separately.

In most developed countries, collaborative care is used in various settings, including the primary health care level, to manage the comorbidity of depression and chronic NCDs such diabetes, hypertension and certaint ypes of cancers, and has been proven cost effective for this purpose (13-15). A randomised controlled trial in 14 primary care clinics in Washington State studied patients with depression and poorly controlled diabetes, or coronary heart disease, or both, and concluded that, compared with care as usual, collaborative care led to significant improvement in the management of depression and chronic diseases (32).

Ali et al. (35) and Carney \& Freedland (36) argued that management of chronic noncommunicable disorders based solely on the treatment of a single diagnosis is flawed from the public health perspective. This is because these chronic diseases are usually accompanied by variousco-morbidities such as hypertension, cardiovascular disease and, most commonly, depression, resulting in competing demands when patients seek care from different clinicians.

In conclusion, our finding on absence of protocols or interventions to manage the comorbidity of depression and chronic NCDs means that patients with this co-morbidity continue to receive separated treatments that can have serious health consequences for them. We therefore suggest that there should be a shift from separate treatment of this co-morbidity to collaborative treatment in line with WHO's recommendations. This could be achieved by forming the collaborative care team constituted by a medical doctor, a mental health nurse and a registered nurse based at the district hospital.

\section{REFERENCES}

1. Beaglehole R, Horton R. Chronic diseases: global action must match global evidence. The Lancet. 2010;376(9753):1619-21.

2. Alwan A, MacLean DR, Riley LM, d'Espaignet ET, Mathers CD, Stevens GA, et al. Monitoring and surveillance of chronic non-communicable diseases: progress and capacity in high-burden countries. The Lancet. 2010;376(9755):1861-8.

3. Narayan KV, Ali MK, Koplan JP. Global noncommunicable diseases-where worlds meet. New England Journal of Medicine. 2010;363(13):1196-8.

4. Barghouti F, Yasein NA, Mustafa RAB. Depression and co-morbid chronic illnesses in family practice. HealthMED.477.

5. Moussavi S, Chatterji S, Verdes E, Tandon A, Patel V, Ustun B. Depression, chronic diseases, and decrements in health: results from the World Health Surveys. The Lancet. 2007;370(9590):851-8.

6. Chapman DP, Perry GS, Strine TW. The vital link between chronic disease and depressive disorders. Prev Chronic Dis. 2005;2(1):A14.

7. Harpole LH, Williams JW, Olsen MK, Stechuchak KM, Oddone E, Callahan CM, et al. Improving depression outcomes in older adults with comorbid medical illness. General hospital psychiatry. 2005;27(1):412.

8. Bisschop MI, Kriegsman DM, Beekman AT, Deeg DJ. Chronic diseases and depression: the modifying role of psychosocial resources. Social science \& medicine. 2004;59(4):721-33.

9. Noël PH, Williams JW, Unützer J, Worchel $\mathrm{J}$, Lee S, Cornell J, et al. Depression and comorbid illness in elderly primary care patients: impact on multiple domains of health status and well-being. The Annals of Family Medicine. 2004;2(6):555-62.

10. Yang Y-L, Liu L, Wang Y, Wu H, Yang X$\mathrm{S}$, Wang J-N, et al. The prevalence of depression and anxiety among Chinese 
adults with cancer: a systematic review and meta-analysis. BMC cancer. 2013;13(1):393.

11. American Heart Association. Depression and Heart Health 2011 [cited 2012 21st September ]. Available from: http://www.heart.org/HEARTORG/Conditio ns/More/MyHeartandStrokeNews/Depressio n-and-Heart-

Health_UCM_440444_Article.jsp. .

12. Egede LE. Major depression in individuals with chronic medical disorders: prevalence, correlates and association with health resource utilization, lost productivity and functional disability. General hospital psychiatry. 2007;29(5):409-16.

13. Ngo VK, Rubinstein A, Ganju V, Kanellis P, Loza N, Rabadan-Diehl C, et al. Grand challenges: integrating mental health care into the non-communicable disease agenda. PLoS Med 2013;10(5):Page?.

14. 14. Vogeli C, Shields AE, Lee TA, Gibson TB, Marder WD, Weiss KB, et al. Multiple chronic conditions: prevalence, health consequences, and implications for quality, care management, and costs. Journal of general internal medicine. 2007;22(3):391.

15. Katon W. The comorbidity of diabetes mellitus and depression. The American journal of medicine. 2008;121(11):S8-S15.

16. Unützer J, Harbin H, Schoenbaum M, Druss B. The collaborative care model: An approach for integrating physical and mental health care in Medicaid health homes. HEALTH HOME, Information Resource Center. 2013:1-13.

17. Clarke DM, Currie KC. Depression, anxiety and their relationship with chronic diseases: a review of the epidemiology, risk and treatment evidence. Medical Journal of Australia. 2009;190(7):S54.

18. Karinganire K. Non communicable Diseases in Rwanda 2012 [cited 2013 4th September]. Available http://focus.rw/wp/2012/10/mental-healthday-focuses-on-depression/.

19. Speziale HS, Streubert HJ, Carpenter DR. Qualitative research in nursing: Advancing the humanistic imperative: Lippincott Williams \& Wilkins; 2011.
20. Koshy E, Koshy V, Waterman H. Action research in healthcare: Sage; 2010.

21. Elo S, Kyngäs H. The qualitative content analysis process. Journal of advanced nursing. 2008;62(1):107-15.

22. Simon GE. Treating depression in patients with chronic disease. Western Journal of medicine. 2001;175(5):292.

23. Wang PS, Lane M, Olfson M, Pincus HA, Wells KB, Kessler RC. Twelve-month use of mental health services in the United States: results from the National Comorbidity Survey Replication. Archives of general psychiatry. 2005;62(6):629-40.

24. Kessler D, Sharp D, Lewis G. Screening for depression in primary care. British Journal of General Practice. 2005;55(518):659-60.

25. 25. Roundy K, Cully JA, Stanley MA, Veazey C, Souchek J, Wray NP, et al. Are anxiety and depression addressed in primary care patients with chronic obstructive pulmonary disease? A chart review. Primary care companion to the Journal of clinical psychiatry. 2005;7(5):213.

26. Williams Jr JW, Noël PH, Cordes JA, Ramirez G, Pignone $M$. Is this patient clinically depressed? Jama. 2002;287(9):1160-70.

27. Katon W. Epidemiology and treatment of depression in patients with chronic medical illness. Dialogues in clinical neuroscience. 2011;13(1):7.

28. Polsky D, Doshi JA, Marcus S, Oslin D, Rothbard A, Thomas N, et al. Long-term risk for depressive symptoms after a medical diagnosis. Archives of internal medicine. 2005;165(11):1260-6.

29. Kang H-J, Kim S-Y, Bae K-Y, Kim S-W, Shin I-S, Yoon J-S, et al. Comorbidity of Depression with Physical Disorders: Research and Clinical Implications. Chonnam medical journal. 2015;51(1):8-18.

30. Moore MJ, Moore PB, Shaw PJ. Mood disturbances in motor neurone disease. Journal of the neurological sciences. 1998;160:S53-S6.

31. Choi Y, Mayer TG, Williams MJ, Gatchel RJ. What is the best screening test for depression in chronic spinal pain patients? The Spine Journal. 2014;14(7):1175-82. 
32. Katon W, Lin EH, Von Korff M, Ciechanowski P, Ludman EJ, Young B, et al. Collaborative care for patients with depression and chronic illnesses. New England Journal of Medicine. 2010;363(27):2611-20.

33. Pomerantz JM. Screening for Depression in Primary Care.: liggott Publishing, Division of CMP Healthcare Media 2005.

34. NICE. Depression in adults: The treatment and management of depression in adults. NICE guideliine. 2009;90.
35. Ali S, Stone M, Peters J, Davies M, Khunti $\mathrm{K}$. The prevalence of co-morbid depression in adults with Type 2 diabetes: a systematic review and meta-analysis. Diabetic Medicine. 2006;23(11):1165-73.

36. Carney RM, Freedland KE. Depression in patients with coronary heart disease. The American journal of medicine. 2008;121(11):S20-S7. 